\title{
Outcomes of bisphosphonate and its supplements for bone loss in kidney transplant recipients: a systematic review and network meta-analysis
}

Yan Yang ${ }^{1,2+}$, Shi Qiü ${ }^{3+}$, Linghui Deng ${ }^{4}$, Xi Tang ${ }^{1}$, Xinrui $\mathrm{Li}^{1}$, Qiang Wei ${ }^{3}$ and Ping Fu ${ }^{1 *}$ (1)

\begin{abstract}
Background: Mineral bone disease constitutes a common complication of post-kidney transplantation, leading to great disability. As there is no consensus on the optimal treatment for post-kidney transplant recipients (KTRs), we aimed to evaluate the efficacy and safety of bisphosphonate and its combined therapies.

Methods: We incorporated relevant trials to perform a network meta-analysis from direct and indirect comparisons. We searched PubMed, Embase and the CENTRAL and the reference lists of relevant articles up to August 1, 2017, for randomized controlled trials. The primary outcome was bone mineral density (BMD) change at the femoral neck and the lumbar spine.

Results: From a total of 864 citations, 18 randomized controlled trials with a total of 1200 participants were included. Five different regimens were considered. Bisphosphonate plus calcium revealed a significant gain in percent BMD change than calcium alone at the femoral neck (mean difference (MD), 5.83; 95\% credible interval (Crl), 1.61 to 9.27). No significant difference was detected when restricting to absolute terms. At the lumbar spine, bisphosphonate and calcium with or without vitamin D analogs outperformed calcium solely (MD, 0.07; 95\% Crl, 0.00 to $0.13 ; \mathrm{MD}, 0.06 ; 95 \% \mathrm{Crl}, 0.02$ to 0.09 ). Compared to calcium with vitamin $\mathrm{D}$ analogs, adding bisphosphonate was associated with marked improvement (MD, $0.03 ; 95 \% \mathrm{Crl}, 0.00$ to 0.05$)$. Considering percent terms, combination of bisphosphonate with calcium and vitamin $D$ analogs showed greater beneficial effects than calcium alone or with either vitamin D analogs or calcitonin (MD, 10.51; 95\% Crl, 5.92 to 15.34; MD, 5.48; 95\% Crl, 2.57 to 8.42; MD, 6.39; $95 \% \mathrm{Crl}, 0.55$ to 12.89). Both bisphosphonate and vitamin D analogs combined with calcium displayed a notable improvement compared to calcium alone (MD, 7.24; 95\% Crl, 3.73 to 10.69; MD, 5.02; 95\% Crl, 1.20 to 8.84).
\end{abstract} Conclusions: Our study suggested that additional use of bisphosphonate was well-tolerated and more favorable in KTRs to improve BMD.

Keywords: Kidney transplant, Bisphosphonates, Bone mineral density, Network meta-analysis

\footnotetext{
* Correspondence: fupinghx@163.com

+Yan Yang and Shi Qiu contributed equally to this work.

${ }^{1}$ Kidney Research Laboratory, Division of Nephrology, National Clinical

Research Center for Geriatrics, West China Hospital, Sichuan University, No.

37, Guoxue Alley, Chengdu, Sichuan, People's Republic of China610041

Full list of author information is available at the end of the article
}

(c) The Author(s). 2018 Open Access This article is distributed under the terms of the Creative Commons Attribution 4.0 International License (http://creativecommons.org/licenses/by/4.0/), which permits unrestricted use, distribution, and reproduction in any medium, provided you give appropriate credit to the original author(s) and the source, provide a link to the Creative Commons license, and indicate if changes were made. The Creative Commons Public Domain Dedication waiver (http://creativecommons.org/publicdomain/zero/1.0/) applies to the data made available in this article, unless otherwise stated. 


\section{Background}

Since kidney transplantation (KT) became an effective treatment of patients with end-stage renal disease (ESRD), clinicians have paid more attention to complications of kidney transplant recipients (KTRs). Post-transplantation bone disease which can result in serious disabilities and fractures has been observed among a large proportion of KTRs [1]. According to Naylor and colleagues [2], the 5 -year cumulative incidence of fracture ranged from 0.85 to $27 \%$ after KT. Hence, prevention and treatment of bone disorders are of great importance to improve high-quality long-term survival of KTRs.

The etiology of transplant bone disease is multifactorial and most KTRs have preexisting chronic kidney disease-mineral and bone disorders (CKD-MBD) [3]. Apart from these, glucocorticoid-induced suppression of bone formation, calcineurin inhibitors (CNIs) and persistent hyperparathyroidism are the most important risk factors for bone loss [4-6]. Postmenopausal status, prolonged immobilization, duration of CKD stage 5, smoking and presence of diabetes may also contribute to bone loss [4]. The Kidney Disease Improving Global Outcomes (KDIGO) guideline [7] suggested that "vitamin D, calcitriol/alfacalcidol, or bisphosphonates be considered for low BMD patients with stable graft function", but it was derived from the very low quality of evidence.

Previous meta-analyses [8, 9] have demonstrated that bisphosphonates have favorable efficacy on bone mineral density (BMD), but questionable effect on the fracture risk. However, these studies did not examine the effect of co-intervention with calcium and/or vitamin D. Moreover, it is still uncertain that the optimal approach to prevent bone loss and whether it is need to use combined therapy. To obtain a better understanding on this issue, we performed a network meta-analysis (NMA). In this NMA, we systematically reviewed the literature and estimated relative treatment effects for all possible comparisons including bisphosphonates and co-intervention.

\section{Methods}

\section{Search strategy}

This systematic review is performed in keeping with Preferred Reporting Items for Systematic Reviews and Meta-analyses (PRISMA) guideline [10]. A comprehensive search was conducted in PubMed, Embase and the Cochrane Library Central Register of Controlled Trials (CENTRAL) by two independent investigators up to August 1st, 2017. The full search parameters for each database was outlined in Additional file 1. Referenced articles and systematic reviews were screened to maximize inclusion of pertinent data.

\section{Selection criteria}

Only randomized controlled trials (RCTs) comparing bisphosphonate-treated and control groups of adult KTRs were included. The full-text original article with at least one interest outcome was finally involved. Two independent investigators (YY, QS) initially screened the citation titles and abstracts. Studies were excluded because of non-English text, combined transplantation. If duplicate studies from the identical authors were found, the reports were grouped together and only the publication with a complete data was used. Any discrepancies in the study inclusion were resolved by consulting the senior authors (TX).

\section{Data extraction and quality assessment}

The independent reviewers (YY, SQ) used a standardized form to extract information from each eligible study. Data regarding study-, patient- and treatment-related characteristics and outcomes were extracted simultaneously. When relevant information was unclear or needed data was unavailable, attempts were made to obtain eligible data from the first or corresponding author of such studies. We assessed the validity of the NMA through a qualitative appraisal of study designs and methods. We executed the tool recommended by the Cochrane Collaboration to evaluate the risk of bias [11].

\section{Outcomes}

The primary outcome was the BMD change (percent change and absolute change [in $\left.\mathrm{g} / \mathrm{cm}^{2}\right]$ ) at the lumbar spine and the femoral neck after successful KT. The secondary outcomes were overall fractures, all-cause mortality, graft loss, acute renal rejection, adverse events. The fractures occurred during reported follow-up time that identified by radiographs were used to calculate fracture incidence. Graft loss was regarded as a doubling of the baseline serum creatinine level or progressing to ESRD again. We used data from the longest complete follow-up, when the outcomes of different follow-up intervals were reported. If investigators published more than one report addressing the same population, we included the most comprehensive report.

\section{Data synthesis and statistical analysis}

The pair-wise meta-analysis by the random-effects model was performed initially [12]. Results were expressed as mean difference (MD) with $95 \%$ confidence intervals (CI) for continuous outcomes (percent change and absolute change in BMD), while the odds ratio (OR) was used for dichotomous variables (fracture, all-cause mortality, graft loss, acute renal rejection, adverse events). The level of statistical significance was set at $P<0.05$ and all statistical tests were two-sided. The statistical heterogeneity among studies was evaluated by the Cochran's $Q$ test and the $I^{2}$ 
statistic. A $P$ value of 0.05 or less for the Q test or an $\mathrm{I}^{2}$ greater than $50 \%$ was suggestive of substantial study heterogeneity.

We performed random-effects Bayesian network meta-analyses for indirect and mixed comparisons using Markov chain Monte Carlo methods in WinBUGS version 1.4.3 (MRC Biostatistics Unit) [13]. A Bayesian fixed-effect framework was deemed appropriate because of the limited number of studies supporting each edge in the network $[13,14]$. We report the resultant effect as OR or MD with corresponding 95\% credibility intervals (CrIs), which are the Bayesian analogue of 95\% CIs. We estimated the relative ranking probability of each strategy and obtained the hierarchy of competing interventions using rankograms and surface under the cumulative ranking curve (SUCRA) [15]. The SUCRA index ranges between 0 (or $0 \%$ ) and 1 (or 100\%), where the treatments with highest and lowest SUCRA are considered to be the best and worst treatments, respectively.

To assess the presence of the inconsistency, we employed the node-splitting method, excluding one direct comparison at a time and estimating the indirect treatment effect for the excluded comparison. To check the assumption of consistency in the entire network, the design-by-treatment model was conducted [14]. If the total residual deviance and the effective number of parameters $(\mathrm{pD})$ are almost the same, the network consistency is considered to be satisfied. We then performed sensitivity analysis and meta-regressions to explore important network inconsistency.

\section{Quality of evidence}

The quality of evidence was rated according to the Grading of Recommendations, Assessment, Development and Evaluation (GRADE) methodology [16]. In this approach, direct evidence from RCTs starts at high quality and can be downgraded based on the risk of bias, indirectness, imprecision, inconsistency (or heterogeneity) and publication bias to levels of moderate, low and relatively low quality [17].

\section{Results}

\section{Study characteristics}

The PRISMA [10] flowchart depicting the electronic searching process is presented in Fig. 1. There are 864 potentially relevant articles identified through electronic and reference searches. According to title and abstract, 821 publications were excluded after the initial screening. A further 26 studies were excluded because they were not RCT, without available data of interest outcomes and lack of full-text. Overall, 18 RCTs (19 publications) [18-36] involving 1200 participants were included in this NMA. The studies were published between October 1998 and March 2015. The details of the

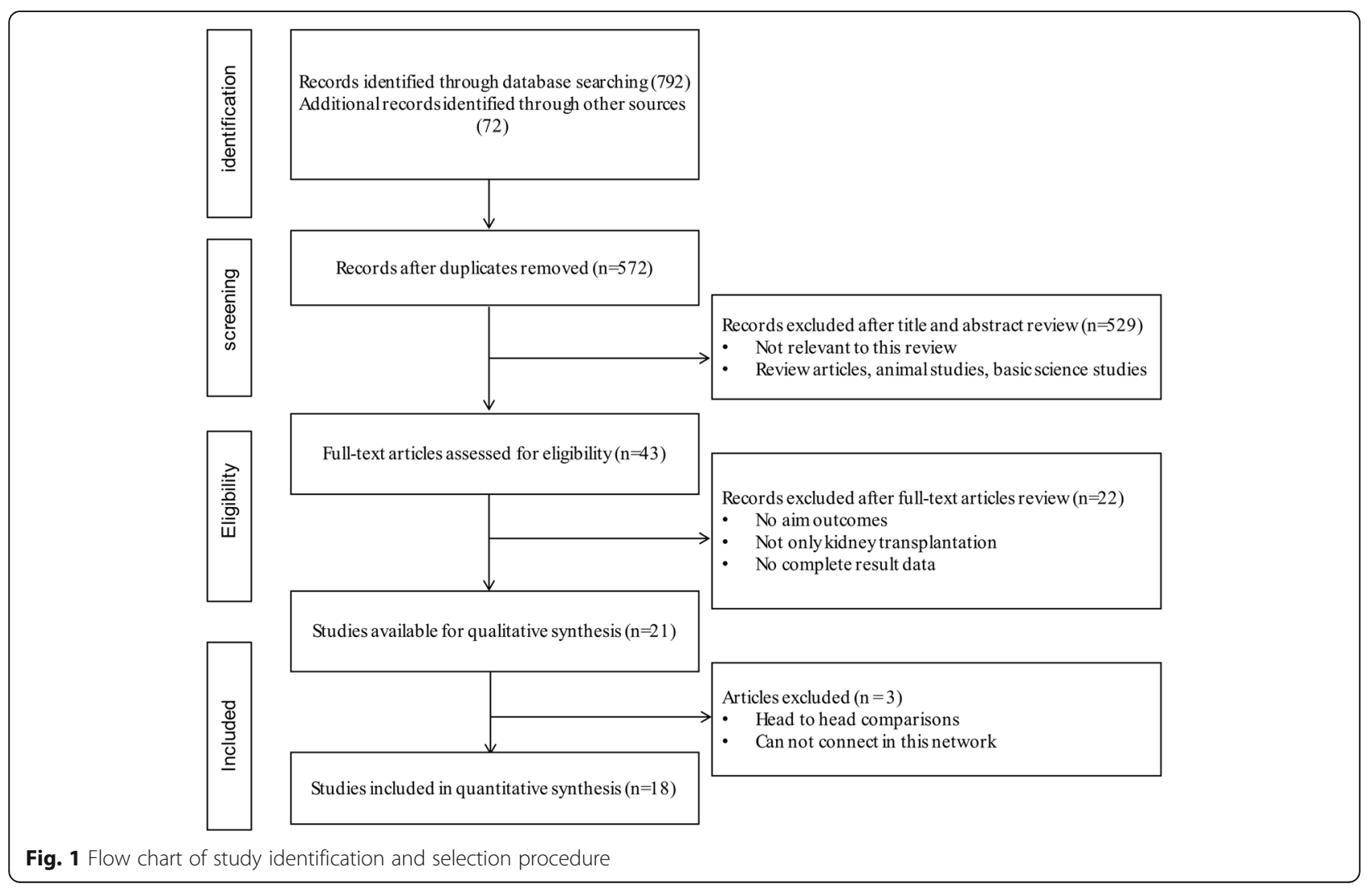


interventions, baseline characteristics of the populations, follow-up period were outlined for NMA in Table 1 . Most of the RCTs included both sexes, except one study [26] only included male patients and two studies [25, 27] did not mention. The number of patients allocated to each treatment ranged from 8 to 66, whereas patient follow-up duration ranged from 6 months to 3 years after first administration.

As expected, most studies compared bisphosphonate with vitamin D analogs (cholecalciferol, alfacalcidol, calcitriol) or placebo. All patients in the trials included received co-intervention including calcium $[24,26-28$, 30-33, 35], vitamin D analogs [19], or both. Bisphosphonate interventions encompassed pamidronate [21, 22, $29,32]$, zoledronic acid [27, 30] and ibandronate [18, 31] that were administered intravenously, while clodronate [33], alendronate [23-26, 28, 34, 35] and risedronate [19, $20,36]$ were given orally.

\section{Risk of Bias assessment result}

The results from the risk of bias assessment are provided in Additional file 2. Details regarding trial methodology were unsatisfactory or incomplete for the majority of studies. Overall, there were 6 (32\%) studies regarded as high risk of bias. Only 10 (53\%) studies performed randomized sequence generation adequately. Furthermore, the risk of bias for concealment of treatment allocation was unclear in 10 (53\%) studies. Only 4 (33\%) studies explicitly reported blinding of participants and investigators, whereas the remaining studies were at high or unclear risk in this regard. The investigators attempted to blind outcome assessors in 6 (32\%) studies, 3 studies did not make an effort to blind assessors, and the residual studies were unclear. When the results were summarized from at least 10 studies, the publication bias accessed via funnel plot. Comparison adjusted funnel plot showed no evidence of asymmetry (Additional file 2).

\section{Pairwise meta-analysis}

Primary results of pairwise meta-analysis (direct comparisons) are summarized in Table 2. In terms of absolute change for the longest follow-up, adding bisphosphonate was associated with a marginal improvement compared to the combination of calcium and vitamin $\mathrm{D}$ analogs (femoral neck: MD, 0.36; 95\% CI, 0.08 to 0.64; lumbar spine: MD, 0.38; 95\% CI, 0.19 to 0.57). Bisphosphonate combined with calcium was significantly better than calcium alone (femoral neck: MD, 1.30; 95\% CI, 0.92 to 1.68; lumbar spine: $\mathrm{MD}, 0.51 ; 95 \% \mathrm{CI}, 0.20$ to 0.82 ). Treatments with calcium alone displayed significantly lower absolute change at the femoral neck than combining with vitamin $\mathrm{D}$ analogs or calcitonin $(\mathrm{MD},-0.74$; 95\% CI, -1.34 to -0.14 ; $\mathrm{MD},-0.55$; $95 \% \mathrm{CI},-1.07$ to $-0.03)$. When measured in percent terms, additional use of vitamin D analogs or bisphosphonate was significantly better than solely calcium (femoral neck: MD 1.53; 95\% CI, 0.88 to 2.18 ; $\mathrm{MD} 1.14 ; 95 \% \mathrm{CI}, 0.78$ to 1.51 ; lumbar spine: MD 2.73; 95\% CI, 1.95 to 3.51 ; MD 1.17; $95 \%$ CI, 0.80 to 1.54$)$. Compared to calcium and vitamin D analogs, the combination of bisphosphonate and calcium showed significant improvement (femoral neck: MD, 1.55; $95 \% \mathrm{CI}, 0.76$ to 2.35 ; lumbar spine: $\mathrm{MD}, 1.53$; $95 \%$ CI, 0.79 to 2.27). Bisphosphonate with calcium and vitamin D analogs also showed a significant gain at the lumbar spine compared to calcium and vitamin D analogs (MD, 1.32; 95\% CI, 1.02 to 1.62).

\section{Network meta-analysis- Primary outcome Change of BMD at the femoral neck}

Ten RCTs involving 536 adults evaluated the absolute change in BMD at the femoral neck. Figure 2 summarizes the network of direct evidence available for this outcome. No statistically significant difference was detected between each treatment groups. The SUCRA value for the regimens were $88 \%, 53 \%, 52 \%, 29 \%, 28 \%$ for bisphosphonate with calcium, bisphosphonate with calcium and vitamin D analogs, calcium with vitamin D analogs, calcitonin with calcium and calcium (Fig. 3a).

The result of percent terms was reported by 5 RCTs including 284 patients. Only bisphosphonate plus calcium revealed a significant gain in percent BMD change than calcium alone (MD, 5.83; 95\% CrI, 1.61 to 9.27). No statistical difference was observed between other groups. Bisphosphonate combined with calcium and vitamin D analogs had the highest SUCRA value (97\% Fig. 3b), followed by calcitonin with calcium (66\%), bisphosphonate plus calcium (38\%), calcium with vitamin D analogs (27\%), and calcium only (22\%).

\section{Change of BMD at the lumbar spine}

14 RCTs including 814 participants provided data for comparison of absolute change in BMD at the lumbar spine. Bisphosphonate and calcium with or without vitamin D analogs outperformed calcium solely (MD, 0.07; 95\% CrI, 0.00 to 0.13 ; MD, 0.06; 95\% CrI, 0.02 to 0.09 ). We also observed that compared to calcium with vitamin $\mathrm{D}$ analogs, adding bisphosphonate was associated with marked improvement (MD, 0.03; 95\% CrI, 0.00 to 0.05). The SUCRA value for each treatment formulations were as follows (Fig. 3c): bisphosphonate with calcium and vitamin D analogs (87\%), bisphosphonate with calcium $(81 \%)$, calcium plus vitamin D analogs (48\%), calcitonin with calcium (24\%) and calcium solely (10\%).

Considering percent terms, the result analyzed using data from 7 trials (466 patients). Combination of bisphosphonate with calcium and vitamin D analogs showed greater beneficial effects than calcium alone or with either vitamin D analogs or calcitonin (MD, 10.51; 


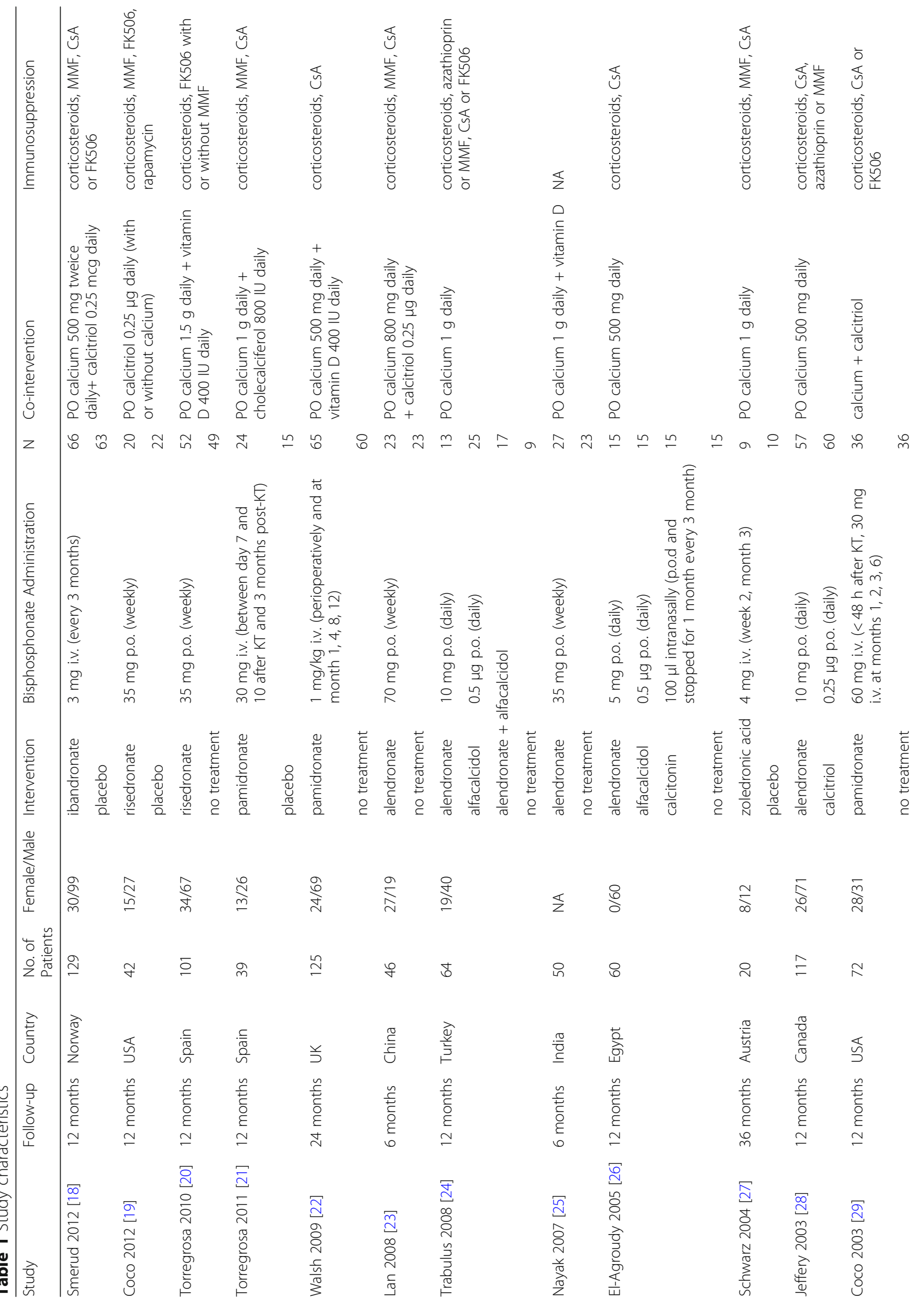


Yang et al. BMC Nephrology

(2018) 19:269

Page 6 of 12

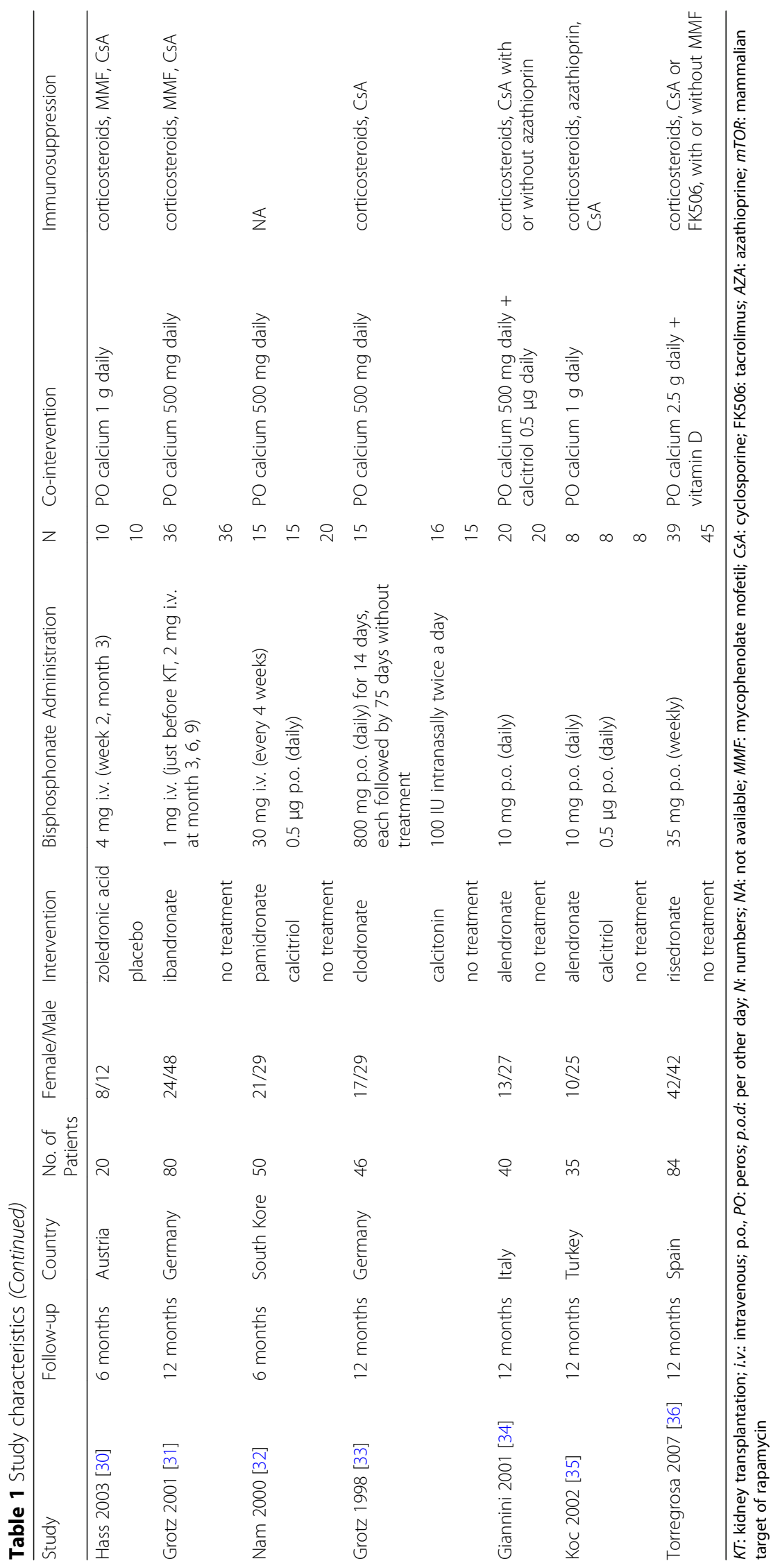


Table 2 Summary effect size of pairwise and network meta-analysis

\begin{tabular}{ll}
\hline Comparisons & $\begin{array}{l}\text { No. of direc } \\
\text { (participants) }\end{array}$ \\
\hline Absolute BMD change at the femoral neck (536) \\
Bis+Ca vs. Bis+Ca + Vit D & $1(29)$ \\
Bis+Ca vs. Ca & $5(167)$ \\
Bis+Ca vs. Ca + Vit D & $2(176)$ \\
Bis+Ca vs. Ca + Calcitonin & $2(61)$ \\
Bis+Ca + Vit D vs. Ca & - \\
Bis+Ca + Vit D vs. Ca + Vit D & $4(206)$ \\
Bis+Ca + Vit D vs. Ca + & - \\
Calcitonin & \\
Ca vs. Ca + Vit D & $2(46)$ \\
Ca vs. Ca + Calcitonin & $2(60)$ \\
Ca + Vit D vs. Ca + Calcitonin & $1(30)$
\end{tabular}

Percent BMD change at the femoral neck (284)

$\begin{array}{ll}\text { Bis+Ca vs. Bis+Ca + Vit D } & 1(29) \\ \text { Bis+Ca vs. Ca } & 4(152) \\ \text { Bis+Ca vs. Ca + Vit D } & 4(46) \\ \text { Bis+Ca vs. Ca + Calcitonin } & 1(31) \\ \text { Bis+Ca + Vit D vs. Ca } & - \\ \text { Bis+Ca + Vit D vs. Ca + Vit D } & - \\ \text { Bis+Ca + Vit D vs. Ca + } & - \\ \text { Calcitonin } & \\ \text { Ca vs. Ca + Vit D } & 3(51) \\ \text { Ca vs. Ca + Calcitonin } & 1(30) \\ \text { Ca + Vit D vs. Ca + Calcitonin } & 1(30)\end{array}$

$1.14(0.78,1.51)$

$1.55(0.76,2.35)$

$-$

$-$

$-1.53(-2.18,-0.88)$

$-$

$-$

Absolute BMD change at the lumbar spine (814)

$\begin{array}{ll}\text { Bis+Ca vs. Bis+Ca + Vit D } & 1(29) \\ \text { Bis+Ca vs. Ca } & 5(167) \\ \text { Bis+Ca vs. Ca + Vit D } & 4(176) \\ \text { Bis+Ca vs. Ca + Calcitonin } & 2(61) \\ \text { Bis+Ca + Vit D vs. Ca } & 1(30) \\ \text { Bis+Ca + Vit D vs. Ca + Vit D } & 8(484) \\ \text { Bis+Ca + Vit D vs. Ca + } & - \\ \text { Calcitonin } & \\ \text { Ca vs. Ca + Vit D } & 2(46) \\ \text { Ca vs. Ca + Calcitonin } & 2(60) \\ \text { Ca + Vit D vs. Ca + Calcitonin } & -\end{array}$

Percent BMD change at the lumbar spine (466)

$\begin{array}{ll}\text { Bis+Ca vs. Bis+Ca + Vit D } & - \\ \text { Bis+Ca vs. Ca } & 4(152) \\ \text { Bis+Ca vs. Ca + Vit D } & 2(46) \\ \text { Bis+Ca vs. Ca + Calcitonin } & 1(31) \\ \text { Bis+Ca + Vit D vs. Ca } & - \\ \text { Bis+Ca + Vit D vs. Ca + Vit D } & 3(145) \\ \text { Bis+Ca + Vit D vs. Ca + } & - \\ \text { Calcitonin }\end{array}$

$1.17(0.80,1.54)$

$1.53(0.79,2.27)$

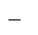$$
-
$$

$1.32(1.02,1.62)$
Network meta-analysis Heterogeneity P-Value Quality of mean differences $(95 \% \mathrm{Crl}) \quad \mathrm{I}^{2}$

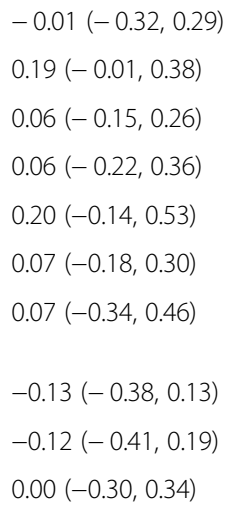

$\begin{array}{lll}- & - & \text { Low } \\ 94.70 \% & 0.000 & \text { Low } \\ 38.10 \% & 0.184 & \text { Moderat } \\ 24.60 \% & 0.249 & \text { Moderat } \\ - & - & \text { Very low } \\ 67.60 \% & 0.026 & \text { Low } \\ - & - & \text { Very low } \\ & & \\ 0.00 \% & 0.403 & \text { Low } \\ 60.20 \% & 0.113 & \text { Low } \\ - & - & \text { Low }\end{array}$

$91.10 \%$

$96.10 \%$

-$$
-
$$$$
-
$$$$
\text { - }
$$

\begin{tabular}{|c|c|}
\hline - & Low \\
\hline 0.000 & Moderate \\
\hline 0.000 & Low \\
\hline- & Low \\
\hline- & Very low \\
\hline- & Very low \\
\hline- & Very low \\
\hline 0.028 & Low \\
\hline- & Low \\
\hline- & Low \\
\hline- & Low \\
\hline 0.571 & Moderate \\
\hline 0.866 & Moderate \\
\hline 0.250 & Moderate \\
\hline- & Low \\
\hline 0.000 & Moderate \\
\hline- & Very low \\
\hline 0.960 & Moderate \\
\hline 0.874 & Moderate \\
\hline- & Very low \\
\hline - & Very low \\
\hline 0.000 & Moderate \\
\hline 0.000 & Low \\
\hline- & Low \\
\hline- & Very low \\
\hline 0.000 & Moderate \\
\hline - & Low \\
\hline
\end{tabular}


Table 2 Summary effect size of pairwise and network meta-analysis (Continued)

\begin{tabular}{|c|c|c|c|c|c|c|}
\hline Comparisons & $\begin{array}{l}\text { No. of directed trials } \\
\text { (participants) }\end{array}$ & $\begin{array}{l}\text { Pairwise meta-analysis mean } \\
\text { differences }(95 \% \mathrm{Cl})\end{array}$ & $\begin{array}{l}\text { Network meta-analysis } \\
\text { mean differences }(95 \% \mathrm{Crl})\end{array}$ & $\begin{array}{l}\text { Heterogeneity } \\
\mathrm{I}^{2}\end{array}$ & $P$-Value & $\begin{array}{l}\text { Quality of } \\
\text { evidence }\end{array}$ \\
\hline Ca vs. Ca + Vit D & $2(51)$ & $-2.73(-3.51,-1.95)$ & $-5.02(-8.84,-1.20)$ & $0.00 \%$ & 0.373 & Moderate \\
\hline Ca vs. Ca + Calcitonin & $1(30)$ & - & $-4.11(-9.01,0.72)$ & - & - & Low \\
\hline $\mathrm{Ca}+$ Vit D vs. Ca + Calcitonin & - & - & $0.91(-4.38,6.44)$ & - & - & Very low \\
\hline
\end{tabular}

Bis = bisphosphonate, $\mathrm{Ca}=$ calcium, Vit $\mathrm{D}=$ Vitamin $\mathrm{D}$ analogs, $95 \% \mathrm{Cl}=95 \%$ Confidence Intervals, $95 \% \mathrm{Crl}=95 \% \mathrm{Credible}$ Intervals. The mean difference with $95 \%$ $\mathrm{Cl}$ or $95 \% \mathrm{Crl}$ was used for continuous outcomes. Significant results are in bold. The Grading of Recommendations Assessment, Development and Evaluation (GRADE) approach specific to NMA served to assess the certainty in the evidence (quality of evidence) associated with specific comparisons, including direct, indirect, and final network meta-analysis estimates. The confidence assessment addressed the risk of bias (in individual studies), imprecision, inconsistency (heterogeneity in estimates of effect across studies), indirectness, and publication bias

95\% CrI, 5.92 to $15.34 ; \mathrm{MD}, 5.48$; $95 \% \mathrm{CrI}, 2.57$ to 8.42 ; MD, 6.39; $95 \%$ CrI, 0.55 to 12.89). Both bisphosphonate and vitamin $\mathrm{D}$ analogs combined with calcium displayed a notable improvement compared to calcium alone (MD, 7.24; 95\% CrI, 3.73 to 10.69 ; MD, 5.02; 95\% CrI, 1.20 to 8.84$)$. As expected, bisphosphonate combined with calcium and vitamin D analogs had the highest SUCRA value (Fig. 3d 99\%), followed by bisphosphonate with calcium $(63 \%)$, calcium with vitamin
D analogs (57\%), calcitonin with calcium (29\%), and calcium only $(2 \%)$.

\section{Secondary outcomes}

We did not observe a significant difference in the incidence of fractures from the direct comparisons and it could not connect to draw network geometries. All treatments have uncertain effects on all-cause mortality and graft loss metrics. Similarly, there were no statistical

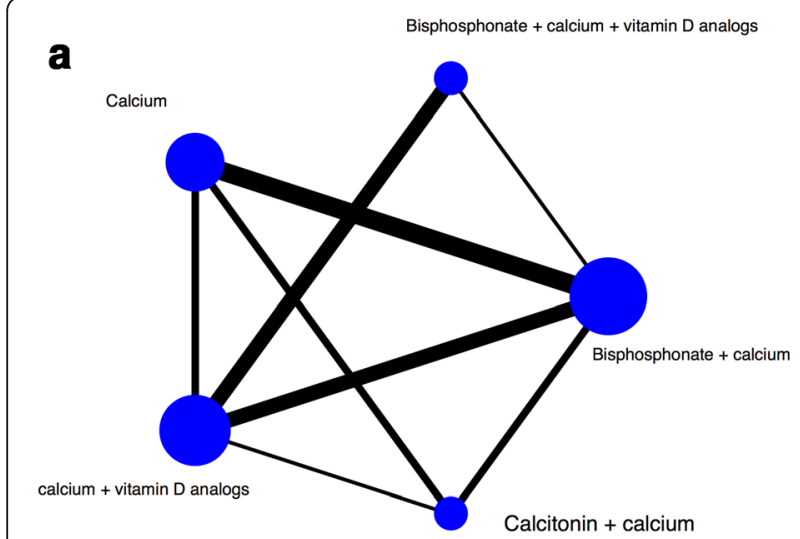

C

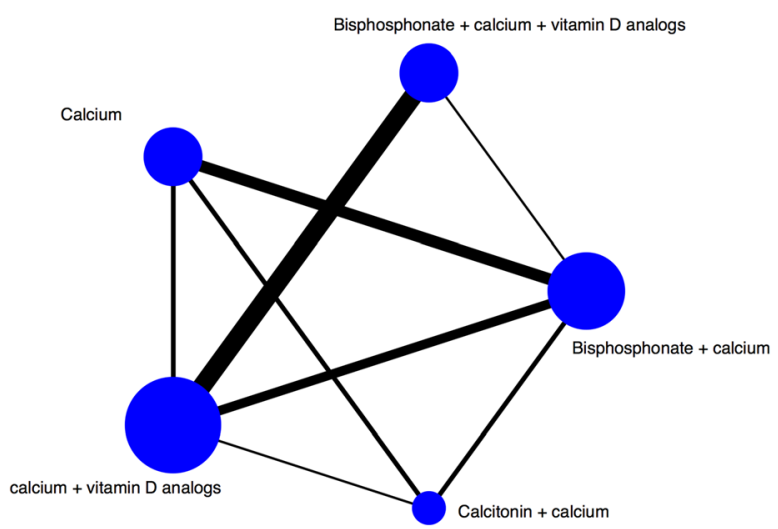

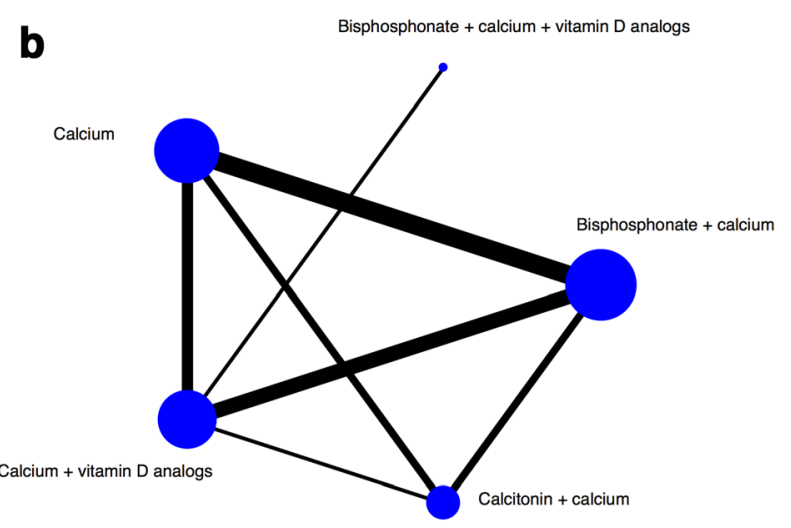

d

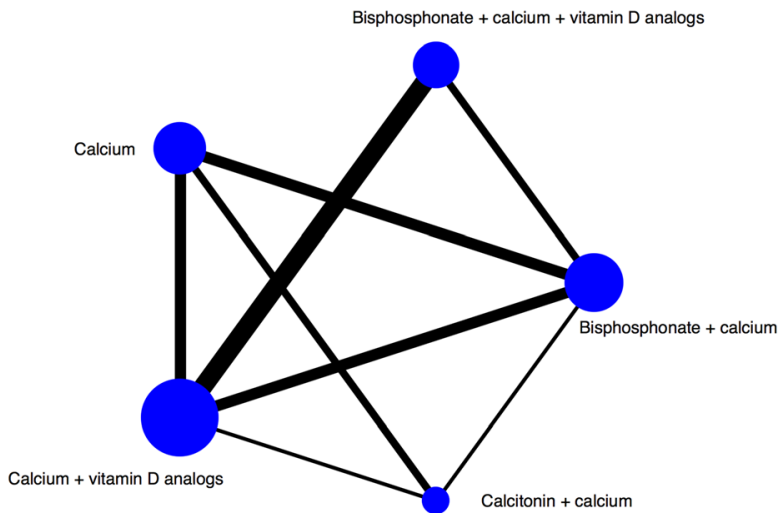

Fig. 2 Network of eligible comparisons for primary outcome. The width of the lines is proportional to the number of trials comparing every pair of treatments, and the size of every circle is proportional to the number of randomly assigned participants (sample size). Network of included studies for all other outcomes is shown in Additional file 3. a Network of absolute change of BMD at the femoral neck; $\mathbf{b}$ Network of percent change of BMD at the femoral neck; c Network of absolute change of BMD at the lumbar spine; (d) Network of percent change of BMD at the lumbar spine. 


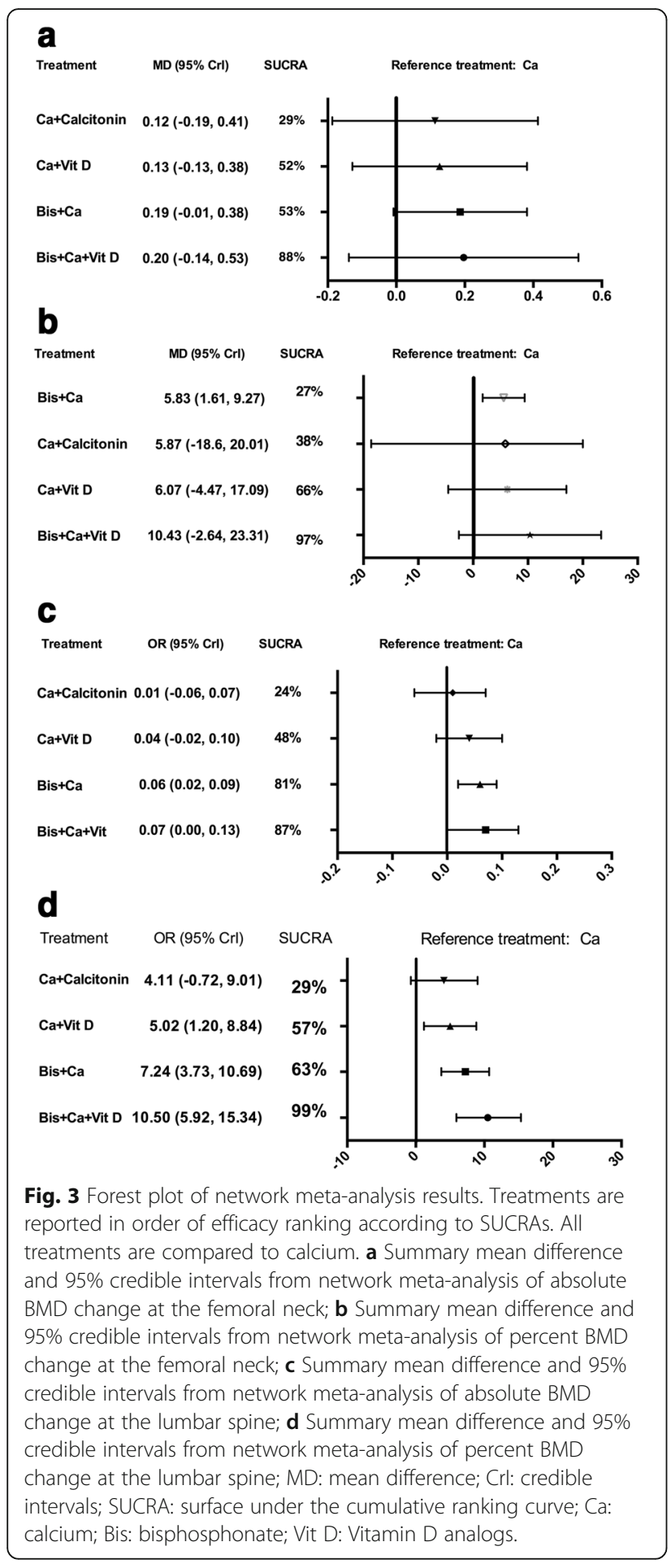

differences in the number of biopsy-proven acute rejections as well as adverse events among treatment groups. However, we found more adverse events happened in bisphosphonate and calcium than in calcium alone (OR, 5.41; 95\% CrI, 1.15 to 25.49) from pairwise meta-analysis. Further details of the secondary outcome analyses are presented in Additional files 3 and 4.

\section{Network consistency}

No evidence of small study effects based on funnel plot asymmetry was observed, but the number of studies included in each comparison was small. There was no evidence of inconsistency in the NMA when we applied the node-splitting approach. The total residual deviance for the outcomes of percent change $(23.73, \mathrm{pD}=22)$ and absolute change $(43.86, \mathrm{pD}=45)$ of $\mathrm{BMD}$ at the lumbar spine implied a good model fit, as well as percent change $(25.22, \mathrm{pD}=26)$ and absolute change (24.36, $\mathrm{pD}=24)$ at the femoral neck.

\section{Sensitivity analysis}

For the sensitivity analyses, we used the full network for the primary outcome. In the first analysis, we investigated the different assumptions regarding the potential relationship between time and treatment effect, Bayesian NMA were repeated using the absolute change of BMD at the twelve-month follow-up period. We observed comparable results at the lumbar spine, adding bisphosphonate showed significant improvement than calcium alone or calcium with vitamin $\mathrm{D}$ analogs (Additional file 5: $\mathrm{MD}$, 0.06; 95\% CrI, 0.01 to 0.10 ; MD, 0.03 ; $95 \%$ CrI, 0.00 to 0.07). We also observed that bisphosphonate with calcium and vitamin $\mathrm{D}$ analogs outperformed calcium solely (MD, 0.07; 95\% CrI, 0.01 to 0.15). At the femoral neck, bisphosphonate with calcium showed a significant preference than calcium alone (Additional file 5; MD, 0.23; 95\% CrI, 0.02 to 0.46). The parameter estimates were consistent with the main analysis. We carried out separate meta-regressions to test the effect of length of trial, publication date and modes of administration. No evidence exists for an interaction between any of the trial characteristics assessed and the treatment effect.

\section{Quality of evidence}

In general, there was no serious risk of bias, indirectness, inconsistency, or publication bias for any of the direct comparisons. In several comparisons, there was serious imprecision in summary estimate because the 95\% credible interval crossed unity. The GRADE quality of evidence supporting the use of each treatment for the primary outcome was outlined in Table 2. According to GRADE, we had moderate confidence in estimates supporting the combination use of bisphosphonate or vitamin $\mathrm{D}$ analogs with calcium for improving BMD at the lumbar spine. We detected using bisphosphonate combined with vitamin $\mathrm{D}$ analogs and calcium considering BMD change at the lumbar spine with low quality evidence. There was very confidence in estimates supporting using calcitonin with calcium both at the lumbar spine and the femoral neck. Conceptually, there was no significant intransitivity. 


\section{Discussion}

This NMA was aimed to investigate the comparable efficacy and safety of bisphosphonate and its co-interventions for the post-transplantation bone disease. We found the combination of bisphosphonate, calcium and vitamin D analogs was the most effective to prevent bone and restore or improve BMD. However, the effects on fracture risk, adverse events, death, acute renal rejection and graft loss were still uncertain because of insufficient data and short follow-up time.

The current study revealed that only calcium prescription could not benefit KTRs from bone loss. We may suggest KTRs take calcium and vitamin D analogs orally because this NMA showed combination therapy of calcium and vitamin D analogs could improve BMD than calcium alone with moderate quality evidence. The result was supported by previous studies $[37,38]$. Our work displayed that calcitonin with calcium seemed only better than calcium alone with low strength of evidence. Two RCTs [26, 33] involving 31 patients allocated to receive calcitonin, and incidence of hypocalcemia was reported so that we did not suggest giving calcitonin for KTRs. In our study, additional use of bisphosphonate could improve BMD changes at the lumbar spine and femoral neck. It was in accordance with previous analyses [9, 39], though they did not examine the effect of calcium and vitamin D analogs supplementation. Importantly, the validity and robustness of NMA depends not only on the heterogeneity in case of standard pairwise meta-analysis, but also on the inconsistency between direct and indirect contrast estimates. No evidence of inconsistency was found in this NMA. Bisphosphonate plus calcium revealed a significant gain in percent BMD change than calcium alone. The heterogeneity was calculated from the pairwise meta-analysis with four RCTs [31-33, 35]. The sample size, races and bisphosphonates which included ibandronate [31], pamidronate [32], clodronate [33], alendronate [35] were different. These resulted in high heterogeneity which would reduce the quality of evidence. Adding bisphosphonates also showed significant improvement than combination of calcium and vitamin D analogs in both absolute and percent BMD change at the lumbar with high heterogeneity. The heterogeneity was associated with characteristics of samples, different inclusion and exclusion criterion. The quality of evidence downgraded because of high heterogeneity. At this stage, limited information made it difficult to perform further analysis. Thus, we could not ignore the impact of heterogeneity when draw the conclusion. Although bisphosphonate with calcium and vitamin $\mathrm{D}$ analogs ranked the best, we did not detect any significant differences between combination use of bisphosphonate and calcium with or without vitamin D analogs from the indirect comparisons. Moreover, indirect comparisons would lead to very low quality of evidence. Only RCT conducted by Fan SL et al. [40, 41] compared bisphosphonate alone with placebo. They found that only two intravenous doses of pamidronate can protect the skeleton from bone loss even 4 years later after transplantation. We could not specify the influence of bisphosphonate monotherapy and access the situation of KTRs with no treatment due to lack of relevant studies.

Included RCTs used BMD as a surrogate marker and did not provide sufficient data to make a polygonal network configuration about the fracture. Also, the association between BMD metrics and fracture risk in KTRs is still controversial. West SL et al. [42] indicated that low BMD was a risk factor for subsequent fracture in patients with pre-dialysis CKD, but data for KTRs are scant. According to KDIGO guideline [7], bone biopsy is reasonable to guide treatment in the first twelve-months after transplantation. However, it is an invasive procedure and most centers lack the expertise to properly process and analyze bone biopsy specimens. Recently, the Fracture Risk Assessment Tool (FRAX) [43] and the spine Trabecular bone score (TBS) [44] were detected as new measurements for KTRs to predict fracture risk. Consequently, surrogate outcomes poorly reflect pathological bone changes. Future trials need to find more specific measurements for detecting mineral and bone disorders in KTRs.

Evidence on other secondary outcomes was limited. There was an unexpected finding from previous reviews $[9,45]$ that bisphosphonate reduced acute graft rejection moderately. Bisphosphonates could suppress cytokine releasing from activated macrophages to inhibit $\mathrm{T}$-cell function. Its immunomodulatory and anti-inflammatory properties may explain this finding. However, the confidence intervals were wide and ignored the influence of co-interventions. The use of bisphosphonate was limited on account of its nephrotoxicity and development of the adynamic bone disease. We did not find additional bisphosphonate use would increase the occurrence of graft loss and adverse events. Apart from mild gastrointestinal side effects, include RCTs did not report or systematically study serious adverse events. Perazella MA et al. [46] summarized that bisphosphonate nephrotoxicity is infusion time-dependent and dose-dependent. Increasing the time interval between doses can limit its nephrotoxicity. On the current situation, bisphosphonate therapy was well tolerated whereas controversial data on its potency in preventing fracture limited its widespread.

Our analysis updated the previous meta-analysis and conducted a comprehensive search with broad inclusion criteria to maximize available data in this field. Only RCTs that supplied BMD results with $\mathrm{g} / \mathrm{cm}^{2}$ were included to standardize each comparison, while some studies used different units such as Z-score or T-score. Furthermore, we used GRADE approach to measure the quality of evidence 
and also performed sensitivity analyses to demonstrate the robustness of estimates. In addition, only adult KTRs were included to offer more reliable evidence and minimize potential bias. To our knowledge, this is the first NMA that took co-intervention (calcium, vitamin $\mathrm{D}$ analogs) into account when examining the effect of bisphosphonates and expands on previous meta-analyses as well $[9,45]$.

However, this NMA still has several limitations including the omission of important methodological details in RCTs and the possibility of reporting biases. Most included studies had a high risk of bias and their impact on results is uncertain. Moreover, some studies only included cadaveric allograft, while some studies excluded patients with diabetes or postmenopausal women. These were risk factors for fracture. Preexisting CKD-MBD, immunosuppression therapy including steroid dosage, CNIs type could also cause bone disease after KT. Because of insufficient information, we could not perform further analysis to identify the influence of relevant factors. These would result in high heterogeneity which may downgrade the quality of evidence as well. Aside from different basic characteristic of the participant, it is unknown if within the drug class of bisphosphonates certain drugs are more favorable than others, and the bisphosphonates regimen (dosage, route, timing, and administration duration) differ among the included studies. We grouped vitamin D, calcitriol, alfacalcidol together as vitamin $\mathrm{D}$ analogs and did not distinguish their efficacy. These factors may potentially influence the calculation of BMD between RCTs.

More high-quality RCT is required to determine the optimal therapy for KTRs to prevent fractures with minimal risk for side effects. We also need to find a more correlative measurement than BMD to reflect pathological bone changes in KTRs. Future studies should be powered to show the fracture risk with sufficient follow-up time ( $\geq 3$ years) and adequate sample sizes, while providing methodological details.

\section{Conclusion}

At this stage, we suggested the additional use of bisphosphonate was well-tolerated and more favorable in KTRs to improve BMD at the lumbar spine and femoral neck. However, evidence to reduce fracture risk is insufficient. Clinicians should take all known safety information and compliance of patients into account when using bisphosphonates. Further studies are needed to support our findings and find optimal treatment option for KTRs.

\section{Additional file}

Additional file 1: Search algorithms. (DOCX $17 \mathrm{~kb})$
Additional file 2: Risk of bias assessments within studies. (DOCX $675 \mathrm{~kb}$ ) Additional file 3: Network plot for secondary outcomes. (DOCX $223 \mathrm{~kb}$ ) Additional file 4: Network meta-analysis of secondary outcomes. (DOCX $22 \mathrm{~kb}$ )

Additional file 5: Subgroup analysis and sensitivity analysis. (DOCX $17 \mathrm{~kb}$ )

\section{Acknowledgments}

The authors would like to acknowledge lan Charles Tobias for reviewing the manuscript.

\section{Funding}

This study was supported by the National Natural Science Foundation of China (Grant No.81570668).

\section{Availability of data and materials}

The datasets used and/or analyzed during the current study are available from the corresponding author on reasonable request.

\section{Authors' contributions}

PF and QW were responsible for the conception and design of the study. YY, SQ did the analysis and interpreted the analysis in collaboration with XRL and LHD. YY, SQ were responsible for the acquisition of data. YY, SQ wrote the first draft of the manuscript. $Y Y, X T$ interpreted the data and wrote the final version. All authors critically revised the Article for important intellectual content and approved the final version. PF and QW obtained public funding.

Ethics approval and consent to participate

All analyses were based on previous published studies, thus no ethical approval and patient consent are required.

Consent for publication

Not applicable.

\section{Competing interests}

The author declare that they have no competing interests.

\section{Publisher's Note}

Springer Nature remains neutral with regard to jurisdictional claims in published maps and institutional affiliations.

\section{Author details}

${ }^{1}$ Kidney Research Laboratory, Division of Nephrology, National Clinical Research Center for Geriatrics, West China Hospital, Sichuan University, No. 37, Guoxue Alley, Chengdu, Sichuan, People's Republic of China610041. 'Department of Nephrology, The First People's Hospital of Changzhou, The Third Affiliated Hospital of Soochow University, Changzhou, Jiangsu, People's Republic of China213000. ${ }^{3}$ Department of Urology, Institute of Urology, West China Hospital, Sichuan University, Chengdu, Sichuan, People's Republic of China. ${ }^{4}$ Stroke Clinical Research Unit, Department of Neurology, West China Hospital, Sichuan University, Chengdu, Sichuan, People's Republic of China.

Received: 2 October 2017 Accepted: 5 October 2018

Published online: 19 October 2018

References

1. Mitterbauer C, Oberbauer R. Bone disease after kidney transplantation. Transpl Int. 2008;21(7):615-24.

2. Naylor KL, Li AH, Lam NN, Hodsman AB, Jamal SA, Garg AX. Fracture risk in kidney transplant recipients: a systematic review. Transplantation. 2013; 95(12):1461-70

3. Weisinger JR, Carlini RG, Rojas E, Bellorin-Font E. Bone disease after renal transplantation. Clin J Am Soc Nephrol. 2006;1(6):1300-13.

4. Bouquegneau A, Salam S, Delanaye P, Eastell R, Khwaja A. Bone disease after kidney transplantation. Clin J Am Soc Nephrol. 2016;11(7):1282-96.

5. Cunningham J. Pathogenesis and prevention of bone loss in patients who have kidney disease and receive long-term immunosuppression. J Am Soc Nephrol. 2007;18(1):223-34.

6. Evenepoel P, Cooper K, Holdaas H, Messa P, Mourad G, Olgaard K, Rutkowski B, Schaefer H, Deng H, Torregrosa JV, Wuthrich RP, Yue S. A 
randomized study evaluating cinacalcet to treat hypercalcemia in renal transplant recipients with persistent hyperparathyroidism. Am J Transplant. 2014;14(11):2545-55

7. Ketteler M, Block GA, Evenepoel P, Fukagawa M, Herzog CA, McCann L, Moe SM, Shroff R, Tonelli MA, Toussaint ND, Vervloet MG, Leonard MB. Executive summary of the $2017 \mathrm{KDIGO}$ chronic kidney disease-mineral and bone disorder (CKD-MBD) guideline update: what's changed and why it matters. Kidney Int. 2017:92(1):26-36.

8. Palmer SC, McGregor DO, Strippoli GF. Interventions for preventing bone disease in kidney transplant recipients. Cochrane Database Syst Rev. 2007.

9. Versele EB, Van Laecke S, Dhondt AW, Verbeke F, Vanholder R, Van Biesen W, Nagler EV. Bisphosphonates for preventing bone disease in kidney transplant recipients: a meta-analysis of randomized controlled trials. Transpl Int. 2015;29(2):153-64.

10. Hutton B, Salanti G, Caldwell DM, Chaimani A, Schmid CH, Cameron C, loannidis JP, Straus S, Thorlund K, Jansen JP, Mulrow C, Catalá-López F, Gøtzsche PC, Dickersin K, Boutron I, Altman DG, Moher D. The PRISMA extension statement for reporting of systematic reviews incorporating network meta-analyses of health care interventions: checklist and explanations. Ann Intern Med. 2015;162(11):777-84.

11. Higgins $G$, Green S. Cochrane handbook for systematic reviews of interventions version 5.1.0. Updated march, 2011. London: The Cochrane Collaboration; 2011.

12. DerSimonian R, Laird N. Meta-analysis in clinical trials. Control Clin Trials. 1986;7:177-88.

13. Salanti G, Higgins JP, Ades AE, loannidis JP. Evaluation of networks of randomised trials. Stat Methods Med Res. 2008;17(3):279-301.

14. Higgins JP, Jackson D, Barrett JK, Lu G, Ades AE, White IR. Consistency and inconsistency in network meta-analysis: concepts and models for multi-arm studies. Res Synth Methods. 2012;3(2):98-110.

15. Higgins JP, Thompson SG, Deeks JJ, Altman DG. Measuring inconsistency in meta-analyses. BMJ. 2003:327(7414):557-60.

16. Guyatt GH, Oxman AD, Vist GE, Kunz R, Falck-Ytter $Y$, Alonso-Coello P, Schünemann HJ, GRADE Working Group. GRADE: an emerging consensus on rating quality of evidence and strength of recommendations. BMJ. 2008; 336(7650):924-6.

17. Salanti G, Del Giovane C, Chaimani A, Caldwell DM, Higgins JP. Evaluating the quality of evidence from a network meta-analysis. PLoS One. 2014;9(7):e99682.

18. Smerud KT, Dolgos S, Olsen IC, Åsberg A, Sagedal S, Reisæter AV, Midtvedt K, Pfeffer P, Ueland T, Godang K, Bollerslev J, Hartmann A. A 1-year randomized, double-blind, placebo-controlled study of intravenous ibandronate on bone loss following renal transplantation. Am J Transplant. 2012;12(12):3316-25

19. Coco M, Pullman J, Cohen HW, Lee S, Shapiro C, Solorzano C, Greenstein S, Glicklich D. Effect of risedronate on bone in renal transplant recipients. J Am Soc Nephrol. 2012;23(8):1426-37.

20. Torregrosa JV, Fuster D, Gentil MA, Marcen R, Guirado L, Zarraga S, Bravo J, Burgos D, Monegal A, Muxí A, García S. Open-label trial: effect of weekly risedronate immediately after transplantation in kidney recipients. Transplantation. 2010;89(12):1476-81.

21. Torregrosa JV, Fuster D, Monegal A, Gentil MA, Bravo J, Guirado L, Muxí A, Cubero J. Efficacy of low doses of pamidronate in osteopenic patients administered in the early post-renal transplant. Osteoporos Int 2011:22(1):281-7.

22. Walsh SB, Altmann P, Pattison J, Wilkie M, Yaqoob MM, Dudley C, Cockwell P, Sweny P, Banks LM, Hall-Craggs M, Noonan K, Andrews C, Cunningham J. Effect of pamidronate on bone loss after kidney transplantation: a randomized trial. Am J Kidney Dis. 2009:53(5):856-65.

23. Lan G, Peng L, Xie X, Peng F, Wang Y, Yu S. Alendronate is effective to treat bone loss in renal transplantation recipients. Transplant Proc. 2008;40(10):3496-8.

24. Trabulus S, Altiparmak MR, Apaydin S, Serdengecti K, Sariyar M. Treatment of renal transplant recipients with low bone mineral density: a randomized prospective trial of alendronate, alfacalcidol, and alendronate combined with alfacalcidol. Transplant Proc. 2008;40(1):160-6.

25. Nayak B, Guleria S, Varma M, Tandon N, Aggarwal S, Bhowmick D, Agarwal SK, Mahajan S, Gupta S, Tiwari SC. Effect of bisphosphonates on bone mineral density after renal transplantation as assessed by bone mineral densitometry. Transplant Proc. 2007;39(3):750-2.
26. El-Agroudy AE, El-Husseini AA, El-Sayed M, Mohsen T, Ghoneim MA. A prospective randomized study for prevention of postrenal transplantation bone loss. Kidney Int. 2005;67(5):2039-45.

27. Schwarz C, Mitterbauer C, Heinze G, Woloszczuk W, Haas M, Oberbauer R. Nonsustained effect of short-term bisphosphonate therapy on bone turnover three years after renal transplantation. Kidney Int. 2004;65(1):304-9.

28. Jeffery JR, Leslie WD, Karpinski ME, Nickerson PW, Rush DN. Prevalence and treatment of decreased bone density in renal transplant recipients: a randomized prospective trial of calcitriol versus alendronate. Transplantation. 2003;76(10):1498-502.

29. Coco M, Glicklich D, Faugere $M C$, Burris L, Bognar I, Durkin P, Tellis V, Greenstein S, Schechner R, Figueroa K, McDonough P, Wang G, Malluche $\mathrm{H}$. Prevention of bone loss in renal transplant recipients: a prospective, randomized trial of intravenous pamidronate. J Am Soc Nephrol. 2003; 14(10):2669-76.

30. Haas M, Leko-Mohr Z, Roschger P, Kletzmayr J, Schwarz C, Mitterbauer C, Steininger R, Grampp S, Klaushofer K, Delling G, Oberbauer R. Zoledronic acid to prevent bone loss in the first 6 months after renal transplantation. Kidney Int. 2003;63(3):1130-6.

31. Grotz W, Nagel C, Poeschel D, Cybulla M, Petersen KG, Uhl M, Strey C, Kirste G, Olschewski M, Reichelt A, Rump LC. Effect of Ibandronate on bone loss and renal function after kidney transplantation. J Am Soc Nephrol. 2001; 12(7):1530-7.

32. Nam JH, Moon Jl, Chung SS, Kim SI, Park KI, Song YD, Kim KR, Lee HC, Huh K, Lim SK. Pamidronate and calcitriol trial for the prevention of early bone loss after renal transplantation. Transplant Proc. 2000;32(7):1876.

33. Grotz WH, Rump LC, Niessen A, Schmidt-Gayk H, Reichelt A, Kirste G, Olschewski M, Schollmeyer PJ. Treatment of osteopenia and osteoporosis after kidney transplantation. Transplantation. 1998;66(8):1004-8.

34. Giannini S, D'Angelo A, Carraro G, Nobile M, Rigotti P, Bonfante L, Marchini F, Zaninotto M, Dalle Carbonare L, Sartori L, Crepaldi G. Alendronate prevents further bone loss in renal transplant recipients. J Bone Miner Res. 2001:16(11):2111-7.

35. Koc M, Tuglular S, Arikan H, Ozener C, Akoglu E. Alendronate increases bone mineral density in long-term renal transplant recipients. Transplant Proc. 2002;34(6):2111-3

36. Torregrosa JV, Fuster D, Pedroso S, Diekmann F, Campistol JM, Rubí S, Oppenheimer F. Weekly risedronate in kidney transplant patients with osteopenia. Transpl Int. 2007;20(8):708-11.

37. Elagroudy $A E$, et al. Preventing bone loss in renal transplant recipients with vitamin D. J Am Soc Nephrol. 2003;14(11):2975-9.

38. Josephson MA, Schumm LP, Chiu MY, Marshall C, Thistlethwaite JR, Sprague SM. Calcium and calcitriol prophylaxis attenuates posttransplant bone loss. Transplantation. 2004;78(8):1233-6.

39. Kan SL, Ning GZ, Chen LX, Zhou Y, Sun JC, Feng SQ. Efficacy and safety of bisphosphonates for low bone mineral density after kidney transplantation: a meta-analysis. Medicine. 2016;95(5):e2679.

40. Fan SL, Almond MK, Ball E, Evans K, Cunningham J. Pamidronate therapy as prevention of bone loss following renal transplantation. Kidney Int. 2000; 57(2):684-90.

41. Fan SL, Kumar S, Cunningham J. Long-term effects on bone mineral density of pamidronate given at the time of renal transplantation. Kidney Int. 2003; 63(6):2275-9.

42. West SL, Lok CE, Langsetmo L, Cheung AM, Szabo E, Pearce D, Fusaro M, Wald R, Weinstein J, Jamal SA. Bone mineral density predicts fractures in chronic kidney disease. J Bone Miner Res. 2015;30(5):913-9.

43. Naylor KL, Leslie WD, Hodsman AB, Rush DN, Garg AX. FRAX predicts fracture risk in kidney transplant recipients. Transplantation. 2014;97(9):940-5.

44. Luckman M, Hans D, Cortez N, Nishiyama KK, Agarawal S, Zhang C, Nikkel L, lyer S, Fusaro M, Guo EX, McMahon DJ, Shane E, Nickolas TL. Spine trabecular bone score as an Indicator of bone microarchitecture at the peripheral skeleton in kidney transplant recipients. Clin J Am Soc Nephrol. 2017:12(4):644-52.

45. Palmer SC, Mcgregor DO, Strippoli GF. Interventions for preventing bone disease in kidney transplant recipients: a systematic review of randomized controlled trials. Am J Kidney Dis. 2005:45(4):638-49.

46. Perazella MA, Markowitz GS. Bisphosphonate nephrotoxicity. Kidney Int. 2008:74(11):1385-93. 